\title{
DOES TREATMENT STRATEGY FOR ATRIAL FIBRILLATION INFLUENCE MYOCARDIAL CONTRACTILE FUNCTION?
}

\author{
V.I. Podzolkov, A.I. Tarzimanova*, I.J. Loriya, L.N. Mokhammadi \\ I.M. Sechenov First Moscow State Medical University. Trubetskaya ul. 8-2, Moscow, 119991 Russia
}

Concept of deterioration of myocardial contractile function under long-term intake of antiarrhythmic drugs may be regarded as one of the myths of modern cardiology. Multiple references to negative influence of majority of antiarrhythmic drugs on myocardial inotropic function are speculative. Studies on estimation of influence of arrhythmia treatment strategy on myocardial contractile function are almost absent.

Aim. To estimate dynamics of myocardial contractile function in patients with ischemic heart disease (IHD) and persistent atrial fibrillation (AF) compared among those treated with amiodarone $200 \mathrm{mg}$ daily and with bisoprolol $5 \mathrm{mg}$ daily.

Material and methods. A total of 47 IHD patients with persistent AF were enrolled into the study. Sinus rhythm (SR) was restored during the first 24 hours of hospitalization in all the patients. After SR restoration the patients were randomly allocated to two groups receiving either amiodarone 200 mg daily during 6 months for SR maintenance (group 1) or bisoprolol 5 mg daily for ventricular rate (VR) control (group 2). To estimate myocardial inotropic function all patients underwent steady-state radionuclide ventriculography (RVG) and echocardiography during the first 24 hours after SR restoration and 6 months later.

Results. We revealed changes in left ventricle (LV) diastolic function, reduction of left atrium (LA) contribution to LV diastole and enlargement of LA anterior-posterior dimension during the first 24 hours after SR restoration in the patients of both groups.

6 -month SR maintenance in the first group of patients promoted significant decrease in isovolumic relaxation time (IVRT) from 103.4 $\pm 1.01 \mathrm{~ms}$ to $96.4 \pm 1.1 \mathrm{~ms}$ ( $p=0.02$ ) and reduction of LA anterior-posterior dimension up to $36.1 \pm 3.8 \mathrm{~mm}(p=0.03$ ). Target VR achievement in the second group of patients promoted restoration of LV diastolic function (decrease in IVRT from $104.3 \pm 1.2 \mathrm{~ms}$ to $97.3 \pm 1.2 \mathrm{~ms} ; \mathrm{p}=0.03)$ but did not influence LA size ( $44.1 \pm 3.1 \mathrm{~mm}$ and $43.5 \pm 3.0 \mathrm{~mm}$, respectively). Atrial inotropic function was only changed in the patients of group 1 , the patients of group 2 did not reveal significant change in LA contribution to LV diastole.

Conclusion. 6-month SR maintenance at amiodarone intake in IHD patients with persistent AF resulted in LA contraction, restoration of its contractility and improvement of LV diastolic parameters. Target VR for 6 months of bisoprolol intake led to LV diastolic function improvement, but did not influence LA dimension and contractile function.

Key words: atrial fibrillation; myocardial contractile function; AF treatment tactics; arrhythmogenic remodeling

Ration Pharmacother Cardiol 2013;9(6):619-626

Влияет ли выбор стратегии лечения фибрилляции предсердий на состояние сократительной функции миокарда?

В.И. Подзолков, А.И. Тарзиманова*, И.Ж. Лория, Л.Н. Мохаммади

Первый Московский государственный медицинский университет им. И.М. Сеченова. 119991, Москва, ул. Трубецкая, 8, стр. 2

Одним их существующих мифов современной кардиологии можно считать убеждение большинства клиницистов, что длительное назначение антиаритмических препаратов ухудшает состояние сократительной функции миокарда. Многократные упоминания в литературе о негативном влиянии большинства антиаритмических препаратов на инотропную функцию миокарда делаются гипотетически. Практически нет работ по изучению влияния стратегии лечения аритмии на сократительную функцию сердца.

Цель. Изучить изменение сократительной функции миокарда у больных ишемической болезнью сердца (ИБС) с персистирующей формой фибрилляции предсердий (ФП) при лечении амиодароном в дозе 200 мг/сут в сравнении с терапией бисопрололом в дозе 5 мг/сут.

Материал и методы. В исследование включено 47 пациентов ИБС с персистирующей формой ФП. Всем больным синусовый ритм (СР) был восстановлен в течение первых сут от момента госпитализации. После восстановления СР пациенты были разделены на 2 группы: пациенты I группы для сохранения СР в течение последующих 6 мес принимали амиодарон в дозе 200 мг/сут, больным II группы для контроля частоты желудочковых сокращений (чЖС) был назначен бисопролол в дозе 5 мг/сут. Для оценки инотропной функции миокарда всем больным проводилась равновесная радионуклидная вентрикулография (РРВГ) и эхокардиография в первые сут после восстановления синусового ритма (СР) и через 6 мес лечения.

Результаты. У пациентов I и || группы в первые сут после восстановления СР обнаружены изменения диастолической функции миокарда левого желудочка (Лж), уменьшение показателя вклада левого предсердия (ЛП) в диастолу лЖ и увеличение передне-заднего размера ЛП.

у больных I группы сохранение СР в течение 6 мес способствовало достоверному и значимому уменьшению времени изоволюмического расслабления (ВИР) с 103,4 1,01 мсек

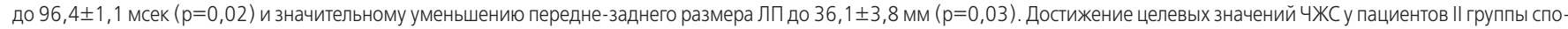

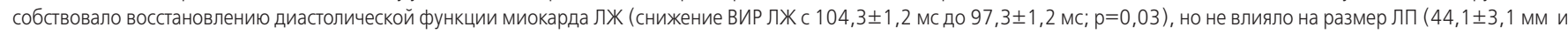

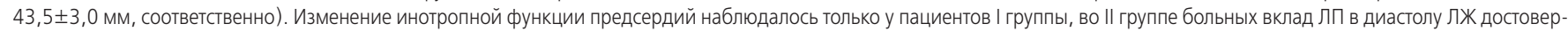
но не изменился.

Заключение. Сохранение синусового ритма в течение 6 мес лечения амиодароном у больных ИБС с персистирующей формой ФП способствовало уменьшению размера ЛП, восстановлению его сократимости и улучшению показателей диастолической функции миокарда ЛЖ. Достижение целевых значений ЧжС на протяжении 6 мес лечения бисопрололом способствовало улучшению показателей диастолической функции миокарда ЛЖ, но не влияло на размер и сократительную функцию Лп. Ключевые слова: фибрилляция предсердий, сократительная функция сердца, выбор тактики лечения ФП, аритмогенное ремоделирование

Рациональная фармакотерапия в кардиологии 2013;9(6):619-626

*Corresponding author (Автор, ответственный за переписку): tarzimanova@mail.ru

\author{
Authors' information: \\ Valery I. Podzolkov - Ph.D., MD, Professor, Head of Faculty Therapy \\ Chair №2, Medical Faculty of the I.M. Sechenov First State Moscow \\ Medical University \\ Aida I. Tarzimanova - Ph.D., MD, Docent of the same chair \\ Irakli Hz. Loria - MD, Ph.D. candidate of the same chair \\ Leila N. Mokhammadi - MD, Ph.D. candidate \\ of the same chair
}

\author{
Сведения об авторах \\ Подзолков Валерий Иванович - Д.м.н., профессор, \\ зав. кафедрой факультетской терапии №2 лечебного \\ факультета Первого МГМУ им. И.М.Сеченова \\ Тарзиманова Аида Ильгизовна - К.М.Н, доцент той \\ же кафедры
}

Лория Иракли Жанович - аспирант той же кафедры Мохаммади Лейла Насировна - аспирант той же кафедры 
Concept of deterioration of myocardial contractile function under long-term intake of antiarrhythmic drugs may be regarded as one of the myths of modern cardiology. Multiply references to negative influence of majority of antiarrhythmic drugs on myocardial inotropic function are speculative. Studies on estimation of influence of arrhythmia treatment strategy on myocardial contractile function are almost absent.

Atrial fibrillation (AF) is on of the most common heart rhythm disturbances in daily clinical practice. AF onset is accompanied by significant subjective symptoms and leads to poor quality of life. AF presence is associated with nearly two-fold mortality incidence rate increase predominantly due to stroke occurrence and chronic heart failure (CHF) progression [1].

There are two main medical AF treatment approaches: cardiac rhythm control and ventricular rate (VR) control [2]. Benefits of VR control tactics are good tolerability and lower incidence of side effects. The drawback of such treatment is a maintenance of arrhythmia symptoms. Sinus rhythm (SR) restoration and retention allows to decrease arrhythmia-associated symptoms, prevents arrhythmogenic myocardial remodeling, reduces risk of thromboembolic complications. However treatment efficacy of majority of antiarrhythmic drugs remains to be very low, while risk of side effects rather high.

Although numerous large-scale randomized trials (PIAF, AFFIRM, RACE, STAF, HOT CAFE, AF CHF) have not revealed prognostic advantage of any of these tactics [3-8], strategy for VR control is recommended as first line therapy for all the AF patients. Antiarrhythmic drugs prescription is only recommended in patients with severe arrhythmia symptoms [2].

However recently pursued experimental and clinical studies demonstrate that arrhythmogenic atrial remodeling occurs at frequent and long-term AF paroxysms [9]. In this case prescription of highly effective antiarrhythmic drugs allows to prevent CHF in AF patients which means the improvement of their survival prognosis.

The aim of the study: to estimate dynamics of myocardial contractile function in patients with ischemic heart disease (IHD) and persistent AF compared among those treated with amiodarone $200 \mathrm{mg}$ daily and with bisoprolol $5 \mathrm{mg}$ daily.

\section{Material and methods}

A total of 47 IHD patients with persistent AF were enrolled into an open-label randomized study. The inclusion criteria were AF paroxysms of not less than
Одним их существующих мифов современной кардиологии можно считать убеждение большинства клиницистов, что длительное назначение антиаритмических препаратов ухудшает состояние сократительной функции миокарда. Многократные упоминания в литературе о негативном влиянии большинства антиаритмических препаратов на инотропную функцию миокарда делаются гипотетически. Практически нет работ по изучению влияния стратегии лечения аритмии на сократительную функцию сердца.

Фибрилляция предсердий (ФП) - наиболее распространенное нарушение сердечного ритма, встречающееся в клинической практике. Появление ФП сопровождается выраженными субъективными расстройствами и ведет к ухудшению качества жизни. Наличие у пациентов ФП ассоциируется с приблизительно двукратным увеличением смертности, в значительной степени обусловленной развитием инсульта и прогрессированием хронической сердечной недостаточности (XCH) [1].

Существуют два основных медикаментозных направления в лечении больных с ФП: контроль ритма и контроль частоты желудочковых сокращений (ЧЖС) [2]. К преимуществам тактики контроля ЧЖС при ФП относятся хорошая переносимость препаратов и меньшая частота побочных эффектов. Недостатком такой тактики лечения является сохранение симптомов аритмии. Восстановление и удержание синусового ритма (СР) у пациентов с ФП позволяет уменьшить симптомы аритмии, предупреждает развитие аритмогенного ремоделирования миокарда, снижает риск появления тромбоэмболических осложнений. Однако эффективность лечения при назначении большинства антиаритмических препаратов остается очень низкой, а риск развития побочных эффектов - достаточно высоким.

Несмотря на то, что в многочисленных, крупных рандомизированных исследованиях (PIAF, AFFIRM, RACE, STAF, HOT (AFE, AF CHF) не было выявлено прогностического преимущества одной тактики лечения над другой [3-8], стратегия контроля ЧЖС рекомендована как терапия первой линии для лечения всех пациентов с ФП. Назначение антиаритмических препаратов официально рекомендовано только тем больным, у которых имеется выраженные симптомы аритмии [2].

Однако, результаты экспериментальных и клинических исследований, проведенные в последние годы, доказывают, что аритмогенное ремоделирование предсердий возникает при появлении частых и длительных пароксизмов ФП [9]. В таком случае, назначение антиаритмических препаратов с высокой эффективностью позволяет предотвратить развитие $\mathrm{XCH}$ у больных с ФП, а значит, улучшает прогноз их выживаемости.

Цель исследования: изучить изменение сократительной функции миокарда у больных ИБС с персистирующей формой ФП при лечении амиодароном в дозе 200 мг/сут в сравнении с терапией бисопрололом в дозе 5 мг/сут. 
48 hours of duration affirmed by ECG or Holter monitoring. The exclusion criteria were the follows: sick sinus syndrome, AV block of II-III degree, long QT syndrome, Wolff-Parkinson-White syndrome, Brugada syndrome; history of myocardial infarction; acute coronary syndrome; chronic heart failure of IIB-III degree by N.D. Stragesko-V.Ch. Vasilenko classification or New York Heart Association (NYHA) functional classes III-IV; inflammatory heart diseases: endocarditis, pericarditis, myocarditis; rheumatic and congenital heart diseases; chronic obstructive lung diseases; severe kidney and liver diseases; anemia, obesity of III degree, any thyroid dysfunction and oncological diseases. All patients had signed informed consent. The study protocol was approved by the local ethics committee.

Sinus rhythm was restored during the first 24 hours of hospitalization in all of the patients. To stop AF paroxysm intravenous infusion of amiodarone was used in 42 (89\%) patients. In 2 (11\%) patients SR has been restored spontaneously. After sinus rhythm restoration the patients were randomly allocated to two groups receiving either amiodarone $200 \mathrm{mg}$ daily during 6 months for SR maintenance (group 1) or bisoprolol $5 \mathrm{mg}$ daily for VR control (group 2).

All patients kept a diary with daily registration of their complaints, heart rate and blood pressure. Every 30 days the patients underwent general clinical examination with analysis of their complaints and objective status. Antiarrhythmic treatment efficacy was evaluated by 24-hour ECG monitoring after 7 days, 3 and 6 months from the start of the treatment.

To estimate myocardial inotropic function steady-state radionuclide ventriculography (RVG) was carried out in all of the patients. The primary assessment was performed in all the patients during the first 24 hours after SR restoration, the second one was performed in those who maintained SR after 6 months of the treatment. The information was registered by the gamma camera BASICAM ("Siemens", Germany) and with the help of domestic system of data collection and processing by the "Rada Gold+" company.

To evaluate the main characteristics of systolic and diastolic functions of left (LV) and right ventricles (RV) the following indices were examined: the ejection fraction (EF), the filling fraction at $1 / 3$ of diastole, the ejection fraction at $1 / 3$ of systole, maximal ejection velocity, mean ejection velocity, maximal filling velocity, end diastolic volume (EDV), end systolic volume (ESV), stroke output (SO), atrium contribution

\section{Материал и методы}

В рандомизированное открытое исследование включено 47 пациентов с ИБС и персистирующей формой ФП. Критерием включения пациентов в исследование было наличие документированного пароксизма ФП продолжительностью не более 48 час, подтвержденного при электрокардиографическом исследовании (ЭКГ) или мониторировании ЭКГ по Холтеру.

Критерии исключения: синдром слабости синусового узла, атриовентрикулярная блокада II-III степени, синдромы длинного интервала QT, Вольфа-Паркинсона-Уайта, Бругады; инфаркт миокарда в анамнезе; острый коронарный синдром; наличие хронической сердечной недостаточности ІІБ-ІІІ стадии по классификации Н.Д. Стражеско-В.Х. Василенко или III-IV функционального класса по классификации НьюЙоркской ассоциации сердца (NYHA); воспалительные заболевания сердца: эндокардиты, перикардиты, миокардиты; ревматические и врожденные пороки сердца; хронические обструктивные заболевания легких; тяжелая патология почек, печени; анемии; ожирение III степени; любые нарушения функции щитовидной железы и онкологические заболевания. Все пациенты подписали добровольное информированное согласие на участие в исследовании. Протокол исследования был одобрен локальным этическим комитетом.

Всем больным СР был восстановлен в течение первых сут от момента госпитализации. Для купирования пароксизма ФП 42 (89\%) пациентам назначалась внутривенная инфузия амиодарона, у 2 (11\%) больных СР восстановился самостоятельно. После восстановления СР пациенты были рандомизированы на 2 группы: пациенты I группы для сохранения СР в течение последующих 6 мес принимали амиодарон (Кордарон таблетки, Санофи) 200 мг (сут, больным II группы для контроля ЧЖС был назначен бисопролол в дозе 5 мг/сут.

Все пациенты вели дневник, в котором ежедневно отмечали жалобы, частоту пульса и артериальное давление. Каждые 30 дней больные проходили общее клиническое обследование, которое включало сбор жалоб, оценку объективного статуса больного. Эффективность антиаритмической терапии оценивалась при проведении суточного мониторирования ЭКГ через 7 дней, 3 и 6 мес от начала лечения.

Для оценки инотропной функции миокарда всем больным проводилась равновесная радионуклидная вентрикулография (РРВГ). Первичное исследование было выполнено всем больным в первые сут после восстановления синусового ритма, повторное исследование проводилось пациентам, сохранившим синусовый ритм через 6 мес лечения. Информация регистрировалась с помощью гамма-камеры BASICAM (фирма «Siemens», ФРГ) и отечественной системы сбора и обработки данных фирмы «Рада Голд+».

Для определения основных характеристик систолической и диастолической функции левого (ЛЖ) и правого желудочков (ПЖ) исследовались следующие показатели: фракция выброса (ФВ), наполнение за 1/3 диастолы, выброс за 
to LV diastole; we also revealed hypokinetic, akinetic and asynchronous zones with graphic presentation of scintigrams.

All the enrolled patients underwent echocardiographic examination on the «Siemens» system (3.74 MHz probe) during the first 24 hours after SR restoration and after 6 months of treatment. At that dimensions of cardiac chambers, valves and intracardiac structures state, indices of myocardial contractile function were estimated. Diastolic function was assesses by the following Doppler echocardiography parameters: early maximum diastolic filling velocity (E peak, $\mathrm{m} / \mathrm{s})$, maximum atrial diastolic filling velocity (A peak, $\mathrm{m} / \mathrm{s}), \mathrm{E} / \mathrm{A}$ ratio and isovolumic relaxation time (IVRT).

The results were processed by the Statistica 6.0 program. Quantitative values were described as arithmetic mean $\mathrm{M}$ and its standard deviation $\pm \sigma$. Statistical analysis was performed using nonparametric Mann-Whitney test. Results were considered significant at $p<0.05$, which agrees with criteria used in medical and biological studies.

\section{Results}

Clinical characteristics of the patients are presented in Table 1.

Analyzed groups were matched by age, gender, severity of IHD, arterial hypertension, CHF and arrhythmia duration.
1/3 систолы, максимальная скорость изгнания, средняя скорость изгнания, максимальная скорость наполнения, конечный диастолический объем (КДО), конечный систолический объем (КСО), ударный объем (УО), вклад предсердия, а также выявлялись зоны гипо-, акинезии, асинхронии с графическим изображением сцинтиграмм.

Эхокардиография (Эхо-КГ) на аппарате «Siemens» (Германия), датчиком с частотой 3,74 МГц была проведена всем больным, включенным в исследование в первые сутки после восстановления синусового ритма и через 6 мес лечения. При этом определялись размеры камер сердца, оценивалось состояние клапанного аппарата, внутрисердечных структур, показатели сократительной способности миокарда. Диастолическую функцию сердца изучали, используя доплерографию, по максимальной скорости раннего диастолического наполнения (пик Е, м/с), максимальной скорости предсердного диастолического наполнения (пик A, $\mathrm{M} / \mathrm{C}$ ), их отношению (Е/A) и времени изоволюмического расслабления (ВИР).

Статистическая обработка полученных результатов проводилась на персональном компьютере с помощью программы Statistica 6.0. Цифровые результаты описывались с помощью средней арифметической М и ее средне-квадратического отклонения $\pm \sigma$. Статистический анализ проводился с использованием непараметрического критерия Манна-Уитни. Достоверными считали результат статистических исследований при вероятности ошибки $p<0,05$, что соответствует критериям, принятым в медико-биологических исследованиях.

Table 1. Clinical and demographic characteristics of the patients of groups 1 and 2

Таблица 1. Клинико-демографическая характеристика больных I и II групп

\begin{tabular}{|c|c|c|}
\hline Parameter / Параметр & Group 1 / I группа & Group 2 / II группа \\
\hline Number of patients / Количество пациентов, n & 25 & 22 \\
\hline Mean age, years / Средний возраст, лет & $56.7 \pm 7.7$ & $59.9 \pm 8.7$ \\
\hline Men / Мужчины, n (\%) & $5(20 \%)$ & $4(18.2 \%)$ \\
\hline Women / Женщины, n (\%) & $20(80 \%)$ & $18(81.8 \%)$ \\
\hline \multicolumn{3}{|l|}{ IHD / ИБС } \\
\hline Angina pectoris FC II / Стенокардия напряжения II ФК, n (\%) & $11(44 \%)$ & $10(45.5 \%)$ \\
\hline Angina pectoris FC III / Стенокардия напряжения III ФК, n (\%) & $14(56 \%)$ & $12(54.5 \%)$ \\
\hline Arterial hypertension / Артериальная гипертония, n (\%) & $21(84 \%)$ & $19(86 \%)$ \\
\hline 1 degree / 1 степень, n (\%) & $5(20 \%)$ & $4(18 \%)$ \\
\hline 2 degree / 2 степень, n (\%) & $12(48 \%)$ & $10(45 \%)$ \\
\hline 3 degree / 3 степень, n (\%) & $4(16 \%)$ & $5(23 \%)$ \\
\hline \multicolumn{3}{|l|}{$\mathrm{CHF} / \mathrm{XCH}$} \\
\hline NYHA FCI / I ФK NYHA, n (\%) & $10(40 \%)$ & $9(41 \%)$ \\
\hline NYHA FC II / II ФK NYHA, n (\%) & $15(60 \%)$ & $13(59 \%)$ \\
\hline AF duration, years / Длительность существования ФП, лет & $5.0 \pm 4.91$ & $5.05 \pm 4.78$ \\
\hline \multicolumn{3}{|l|}{ Last paroxysm duration, hours } \\
\hline Продолжительность последнего пароксизма, часы & $8.5 \pm 3.1$ & $7.8 \pm 3.2$ \\
\hline \multicolumn{3}{|l|}{ p $>0.05$ for all the indices } \\
\hline \multicolumn{3}{|c|}{ IHD - ischemic heart disease, CHF - chronic heart failure, FC - functional class, AF - atrial fibrillation } \\
\hline \multicolumn{3}{|c|}{ p>0,05 для всех показателей } \\
\hline \multicolumn{3}{|c|}{ ИБС - ишемическая болезнь сердца; ФК - функциональный класс; ХСН - хроническая сердечная недостаточность; ФП - фибрилляция предсердий } \\
\hline
\end{tabular}


Mean duration of the last AF paroxysm was $8.5 \pm 3.1$ (from 4 to 18 ) hours in the first group of patients and $7.8 \pm 3.2$ hours in the second one.

Echocardiography revealed normal initial LV EF, LV EDV and LV ESV in the patients of the both groups (Table 2). Intergroup distinctions in these baseline parameters were insignificant.

Patients of the both groups had enlarged left atrium (LA) anterior-posterior dimension, at that significant distinctions between the groups were absent. All the enrolled patients had LV diastolic dysfunction: delayed early diastolic filling velocity (decrease in $\mathrm{E} / \mathrm{A}$ ratio $<1.0)$ and increase in LV IVRT (>100 ms). Mean values of this parameter were comparable in the both groups.

RVG demonstrated changes in the LV diastolic function in patients of the both groups during the first 24 hours after SR restoration, which included reduction in the maximal filling velocity and the filling fraction at $1 / 3$ of diastole. Significant distinctions between the groups were absent (Table 3 ).

LA contribution to LV diastole was decreased significantly during the first 24 hours after SR restoration: $15.9 \pm 4.7 \%$ in the patients of group 1 and $16.7 \pm 4.1 \%$ in the patients of group 2 , what reflected significant loss of the LA contractile function.

17 (68\%) patients of the first group have maintained SR after 6 months of the treatment, 8 (32\%) patients had frequent AF episodes what was the reason for change of therapy tactics and exclusion of these patients from the study. 17 (77\%) of the second group patients treated with bisoprolol $5 \mathrm{mg}$ daily had achieved target VR values, in 4 (18\%) patients of group 2 prescription of combined therapy for tar-

\section{Результаты}

Клиническая характеристика больных представлена в табл. 1.

Исследуемые группы были сопоставимы по возрасту, полу, тяжести ИБС, АГ, ХСН и длительности существования аритмии.

Продолжительность последнего пароксизма ФП находилась в пределах от 4 до 18 час и составила в среднем

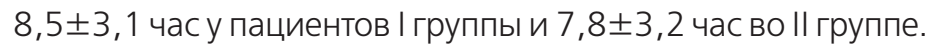

При проведении эхокардиографии в начале исследования было установлено, что все больные сравниваемых групп имели нормальные исходные значения ФВ ЛЖ, КДО Лж и КСО лж (табл. 2). Межгрупповые различия по данным исходным показателям были незначимы.

В I и || группах больных отмечалось увеличение передне-заднего размера левого предсердия (ЛП), при этом значимых различий между группами выявлено не было. У всех пациентов, включенных в исследование, были диагностированы нарушения диастолической функции миокарда ЛЖ в виде замедления заполнения ЛЖ в раннюю диастолу (уменьшение индекса Е/А менее 1,0 ) и увеличения времени изоволюмического расслабления ЛЖ (ВИР более 100 мсек). Средние значения показателя были сопоставимы у пациентов обеих групп.

При проведении РРВГ у пациентов | и || группы в первые сут после восстановления СР обнаружены изменения диастолической функции миокарда ЛЖ в виде уменьшения показателей максимальной скорости наполнения и наполнения за 1/3 диастолы Лж, значимых различий между группами не было (табл. 3).

В первые сутки после восстановления СР было диагностировано значительное уменьшение показателя вклада ЛП

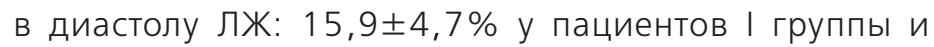
$16,7 \pm 4,1 \%$ во II группе, что отражает значительное снижение сократительной функции ЛП.

Table 2. Dynamics of echocardiographic parameters in the patients of groups 1 and 2

Таблица 2. Динамика показателей эхокардиографии у больных I и II групп

\begin{tabular}{|c|c|c|c|c|}
\hline \multirow[t]{2}{*}{ Parameter / Параметр } & \multicolumn{2}{|c|}{$\begin{array}{c}\text { Group } 1 \text { / I группа } \\
\text { n=25 }\end{array}$} & \multicolumn{2}{|c|}{$\begin{array}{c}\text { Group } 2 \text { / II группа } \\
\text { n=22 }\end{array}$} \\
\hline & $\begin{array}{l}\text { Initially } \\
\text { Исходно }\end{array}$ & $\begin{array}{c}6 \text { months later } \\
\text { Через } 6 \text { мес }\end{array}$ & $\begin{array}{l}\text { Initially } \\
\text { Исходно }\end{array}$ & $\begin{array}{c}6 \text { months later } \\
\text { Через } 6 \text { мес }\end{array}$ \\
\hline LV EF / ФВ ЛЖ,\% & $65.0 \pm 4.5$ & $66.1 \pm 4.6$ & $63.1 \pm 7.3$ & $63.7 \pm 6.7$ \\
\hline LV EDV, ml / КДО ЛЖ, мЛ & $108.1 \pm 28.5$ & $107.6 \pm 27.1$ & $114.1 \pm 30.3$ & $110.4 \pm 28.4$ \\
\hline LV ESV, ml / КСО ЛЖ, мЛ & $37.1 \pm 8.5$ & $36.8 \pm 7.8$ & $41.3 \pm 14.8$ & $39.6 \pm 13.6$ \\
\hline LV MMI, g/m² / ИММ ЛЖ, г/м² & $113.4 \pm 17.2$ & $112.5 \pm 15.7$ & $119.5 \pm 23.2$ & $111.4 \pm 21.7$ \\
\hline \multicolumn{4}{|l|}{ LA anterior-posterior dimension, $\mathrm{mm}$} & $43.5 \pm 3.0$ \\
\hline $\mathrm{E} / \mathrm{A}$ & $0.8 \pm 0.13$ & $0.9 \pm 0.15$ & $0.7 \pm 0.18$ & $0.8 \pm 0.16$ \\
\hline IVRT, ms / BИР, MC & $103.4 \pm 1.01$ & $96.4 \pm 1.1^{*}$ & $104.3 \pm 1.2$ & $97.3 \pm 1.2^{*}$ \\
\hline \multicolumn{5}{|c|}{$\begin{array}{l}\text { *p<0.05 in comparison with initial value in the same group } \\
\text { EF - ejection fraction, LV - left ventricle, EDV - end diastolic volume, ESV - end systolic volume, MMI - myocardial mass index, LA - left atrium, IVRT - isovolumic relaxation tim } \\
\text { *p<0,05 по сравнению с исходным значением В той же группе } \\
\text { ФВ - фракция выброса; ЛЖ - левый желудочек; КДО - конечный диастолический объем; КСО - конечный систолический объем; ИММ - индекс массы миокарда; } \\
\text { ЛП - левое предсердие; ВИР - время изоволюмического расслабления }\end{array}$} \\
\hline
\end{tabular}


Table 3. Dynamics of RVG indices in the patients of groups 1 and 2

Таблица 3. Динамика показателей РРВГ у больных I и II групп

\begin{tabular}{|c|c|c|c|c|}
\hline \multirow[t]{2}{*}{ Parameter / Параметр } & \multicolumn{2}{|c|}{$\begin{array}{c}\text { Group } 1 \text { / I группа } \\
\text { n=25 }\end{array}$} & \multicolumn{2}{|c|}{$\begin{array}{c}\text { Group } 2 \text { / II группа } \\
\text { n=22 }\end{array}$} \\
\hline & $\begin{array}{l}\text { Initially } \\
\text { Исходно }\end{array}$ & $\begin{array}{c}6 \text { months later } \\
\text { Через } 6 \text { мес }\end{array}$ & $\begin{array}{l}\text { Initially } \\
\text { Исходно }\end{array}$ & $\begin{array}{c}6 \text { months later } \\
\text { Через } 6 \text { мес }\end{array}$ \\
\hline HR per min / ЧСС, уд/мин & $68.2 \pm 10.4$ & $70.4 \pm 8.4$ & $72.8 \pm 11.2$ & $72.4 \pm 10.6$ \\
\hline LV EF / ФВ ЛЖ, \% & $63.3 \pm 8.3$ & $63.23 \pm 7.7$ & $67.8 \pm 5.6$ & $66.9 \pm 4.5$ \\
\hline $\begin{array}{l}\text { Atrium contribution to ventricle diastole } \\
\text { Вклад предсердия в диастолу желудочков, \% }\end{array}$ & $15.9 \pm 4.7$ & $21.53 \pm 6.6^{*}$ & $16.7 \pm 4.1$ & $17.4 \pm 4.4$ \\
\hline $\begin{array}{l}\text { Filling fraction at } 1 / 3 \text { of diastole } \\
\text { Наполнение за } 1 / 3 \text { диастолы, \% }\end{array}$ & $20.92 \pm 8.1$ & $27.17 \pm 9.7^{*}$ & $21.3 \pm 7.9$ & $28.5 \pm 11.1^{*}$ \\
\hline $\begin{array}{l}\text { Ejection fraction at } 1 / 3 \text { of systole } \\
\text { Выброс за } 1 / 3 \text { систолы, \% }\end{array}$ & $19.8 \pm 7.8$ & $20.2 \pm 7.6$ & $20.6 \pm 8.5$ & $21.2 \pm 7.9$ \\
\hline $\begin{array}{l}\text { Maximal filling velocity, \%/s } \\
\text { Максимальная скорость наполнения, \%/c }\end{array}$ & $221.4 \pm 71.1$ & $250.7 \pm 76.5^{*}$ & $238.8 \pm 68.6$ & $254.3 \pm 68.4^{*}$ \\
\hline $\begin{array}{l}\text { Maximal ejection velocity, \%/s } \\
\text { Максимальная скорость изгнания, \%/c }\end{array}$ & $316.9 \pm 46.3$ & $310.4 \pm 72.7$ & $330.1 \pm 48.1$ & $340.3 \pm 36.2$ \\
\hline $\begin{array}{l}\text { Mean ejection velocity, \%/s } \\
\text { Средняя скорость изгнания, \%/с }\end{array}$ & $167.8 \pm 32.4$ & $158.9 \pm 31.1$ & $178.1 \pm 20.3$ & $184.6 \pm 37.4$ \\
\hline $\begin{array}{l}\text { *p }<0.05 \text { in comparison with initial value in the same gro } \\
\text { HR - heart rate, EF - ejection fraction, LV - left ventricle } \\
\text { *p }<0,05 \text { по сравнению с исходным значением в той } x \\
\text { чСС - частота сердечных сокращений; ФВ - фракция }\end{array}$ & й желудочек & & & \\
\hline
\end{tabular}

get VR achievement was required, which also was the criterion of exclusion from the study. Myocardial contractile function was estimated repeatedly in 17 (68\%) rhythm-controlled patients who have maintained SR after 6 months of the treatment and in 17 (77\%) rate-controlled patients who have achieved target VR values.

Repeated echocardiography revealed significant improvement in the myocardial diastolic function indices in all the patients (Table 2). Maintenance of SR for 6 months at amiodarone $200 \mathrm{mg}$ /day treatment promoted significant IVRT decrease from 103.4 \pm 1.01 ms to $96.4 \pm 1.1 \mathrm{~ms}(p=0.02)$ and significant reduction in LA anterior-posterior dimension up to $36.1 \pm 3.8 \mathrm{~mm}(p=0.03)$.

Target VR achievement promoted restoration of diastolic function in the second group patients but did not influence LA size. After 6 months of the bisoprolol treatment the patients of group 2 revealed significant IVRT decrease from 104.3 $\pm 1.2 \mathrm{~ms}$ to $97.3 \pm 1.2 \mathrm{~ms}(p=0.03)$. Anterior-posterior LA dimension did not change significantly and made up $44.1 \pm 3.1 \mathrm{~mm}$ and $43.5 \pm 3.0 \mathrm{~mm}$, respectively.

Repeated RVG demonstrated improvement in LV diastolic function parameters in the both groups (Table 3).

SR maintenance at 6 -month amiodarone intake promoted improvement of the LV inotropic function as significant increase in the maximal LV filling velocity from $221.4 \pm 71.1 \% / \mathrm{s}$ to $250.7 \pm 76.5 \% / \mathrm{s}$
Через 6 мес лечения амиодароном СР удержали 17 (68\%) пациентов I группы, у 8 (32\%) больных наблюдались частые эпизоды ФП, что послужило причиной изменения тактики лечения и исключения их из исследования. Целевые значения ЧЖС достигли 17 (77\%) пациентов II группы, которые получали бисопролол в дозе 5 мг/сут, у 4 (18\%) больных для достижения целевых значений чЖС потребовалось назначение комбинированной терапии, что также было критерием исключения пациентов из исследования. Сократительная функция миокарда была оценена повторно у 17 (68\%) больных I группы, сохранивших СР через 6 мес лечения и у 17 (77\%) пациентов II группы, которые достигли целевых значений чЖC.

При повторной Эхо-КГ обнаружено достоверное и значимое улучшение показателей диастолической функции миокарда у всех пациентов (табл. 2). У больных I группы сохранение СР в течение 6 мес лечения амиодароном в дозе 200 мг/сут способствовало достоверному и значимому

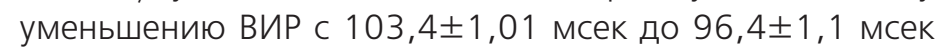
$(p=0,02)$ и значительному уменьшению передне-заднего размера ЛП до 36,1 $\pm 3,8$ мм $(p=0,03)$.

Достижение целевых значений ЧЖС у пациентов II группы способствовало восстановлению диастолической функции миокарда ЛЖ, но не влияло на размер ЛП. Через 6 мес лечения бисопрололом во II группе пациентов наблюдалось достоверное и значимое уменьшение ВИР ЛЖ с 104,3 1 1,2

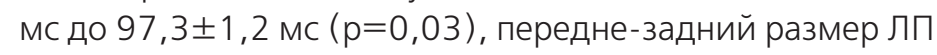

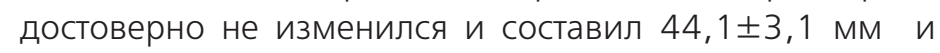
43,5 $\pm 3,0$ мм, соответственно. 
$(p=0.03)$ and the filling fraction at $1 / 3 \mathrm{LV}$ diastole from $20.92 \pm 8.1 \%$ to $27.17 \pm 9.7 \%(p=0.04)$. Repeated RVG in the second group of patients showed similar results. Target VR achievement also promoted improvement of the LV diastolic parameters even if AF paroxysms occurred.

Only group 1 patients demonstrated atrial inotropic function restoration, as LA contribution to LV diastole increased significantly from $15.9 \pm 4.7 \%$ to $21.53 \pm 6.6 \%(p=0.04)$. In the second group patients LA contribution to LV diastole did not change significantly and was $17.4 \pm 4.4 \%$ after 6 months of the treatment. These results confirm that restoration of atrial contractile function is only possible in case of long-term SR maintenance, strategy of VR control does not improve parameters of atrial inotropic function.

\section{Discussion}

At the beginning of the study using echocardiographic and RVG examinations we had revealed LA dilation and decreased atrial contribution to LV diastole. As the assessment of myocardial inotropic function was performed in the first 24 hours after SR restoration we estimated revealed changes as evidence of arrhythmogenic cardiomyopathy. The crucial part in pathogenesis of AF onset and maintenance belongs to alterations of atria anatomic properties such as: atria and pulmonary veins dilation, apoptosis of atrial cardiomyocytes and interstitial fibrosis. Recent experimental studies have demonstrated increased levels of type I angiotensin II receptors and of angiotensin-converting enzyme in tissue of left atrium in patients with $\operatorname{AF}[10,11]$. Stimulation of type I angiotensin II receptors leads to a cascade of phosphorylation reactions and activates different mitogen-activated protein kinases, which results in hypertrophy and apoptosis of atrial cardiomyocytes, accumulation of fibroblasts and collagen induction [12].

Myocardial dyastolic dysfunction has been revealed in all the patients in the first 24 hours after SR restoration. This suggests that high VR during AF episode plays a key role in LV dyastolic dysfunction development [13].

Improvement of LV myocardial diastolic function was revealed after 6 months of the follow-up in all the patients and was independent of AF treatment strategy. Patients of the both groups demonstrated increase in the maximal filling velocity and the filling fraction at $1 / 3$ of LV diastole as well as LV IVRT reduction. Retrospective analysis of the RACE trial has shown that maintenance of the tar-
При проведении контрольной РРВГ наблюдалось улучшение показателей диастолической функции миокарда ЛЖ в I и || группе пациентов (табл. 3).

Сохранение СР при приеме амиодарона в течение 6 мес способствовало улучшению инотропной функции ЛЖ в виде достоверного и значимого увеличения максимальной скорости

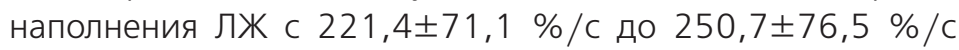

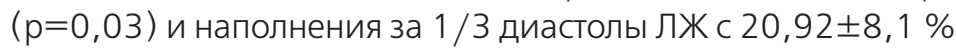
до $27,17 \pm 9,7 \%$ ( $p=0,04)$. Аналогичные результаты были получены при проведении повторной РРВГ у больных II группы. Достижение целевых значений ЧЖС даже при возникновении пароксизмов ФП также способствовало улучшению диастолических показателей Лж.

Восстановление инотропной функции предсердий наблюдалось только у пациентов I группы в виде достоверного и значимого увеличения показателя вклада ЛП в диастолу Лж

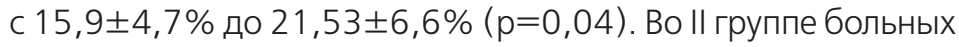
вклад ЛП в диастолу ЛЖ достоверно не изменился и составил $17,4 \pm 4,4 \%$ через 6 мес лечения. Полученные результаты позволяют утверждать, что восстановление сократительной функции предсердий возможно только при условии длительного удержания СР, стратегия контроля ЧЖС не улучшает показателей инотропной функции предсердий.

\section{Обсуждение}

При проведении Эхо-КГ и РРВГ в начале исследования обнаружено увеличение полости ЛП и снижение показателя вклада предсердий в диастолу Лж. Так как изучение инотропной функции миокарда проводилось в первые сут после восстановления СР, выявленные изменения были расценены нами как проявление «аритмогенной» кардиомиопатии. Ведущим звеном в патогенезе развития и поддержания ФП являются изменения анатомических свойств предсердий: дилатация предсердий, расширение устьев легочных вен, апоптоз кардиомиоцитов предсердий, интерстициальный фиброз. Экспериментальные работы последних лет обнаружили увеличение I типа рецепторов ангиотензина II и уровня ангиотензин-превращающего фермента в ткани левого предсердия у пациентов с ФП $[10,11]$. Стимуляция рецепторов ангиотензина II первого типа инициирует каскад процессов фосфорилирования и активирует различные митоген-активируемые протеинкиназы, что приводит к гипертрофии и апоптозу кардиомиоцитов предсердий, аккумуляции фибробластов и индукции коллагена [12].

В первые сут после восстановления СР у всех пациентов была диагностирована диастолическая дисфункция миокарда. Можно полагать, что появление высокой ЧЖС во время эпизода ФП играет главную роль в развитии диастолической дисфункции миокарда Лж [13].

Улучшение показателей диастолической функции миокарда лж было выявлено через 6 мес наблюдения у всех больных и не зависело от выбора стратегии лечения ФП. В I и II группах наблюдалось увеличение максимальной скорости наполнения и наполнение за 1/3 диастолы Лж, а также снижение 
get VR in AF patients for 2-year long follow-up has promoted improvement in LV diastolic function parameters but has not influenced atria size. At that SR maintenance resulted in improvement of LV inotropic function and reduction of LA dimension [14]

LA size reduction and increase in atrium contribution to LV diastole was only observed in those who kept SR for long duration at amiodarone intake. So, early prescription of antiarrhythmic therapy promoted restoration of atrial contractile function and prevented arrhythmogenic atrial remodeling.

\section{Conclusion}

IHD patients with persistent AF during first 24 hours after SR restoration revealed LA dilation with decrease in its contractile function and LV diastolic dysfunction. 6-month bisoprolol treatment with target VR achievement improved parameters of LV diastolic function but did not influence LA size and contractile function. Maintenance of sinus rhythm during 6 months of amiodarone treatment promoted LA size reduction, restoration of its contractility and improvement of LV diastolic function in IHD patients with persistent AF.

Disclosures. All authors have not disclosed potential conflicts of interest regarding the content of this paper.
ВИР ЛЖ. При ретроспективном анализе исследования RACE было показано, что удержание целевых значений ЧЖС у пациентов с ФП на протяжении двухлетнего периода наблюдения способствовало улучшению параметров диастолической функции миокарда ЛЖ, но не влияло на размер предсердий. Сохранение СР приводило к улучшению инотропной функции лж и уменьшению размера ЛП [14]

Уменьшение полости ЛП и увеличение вклада предсердия наблюдалось только у пациентов, длительно удерживающих СР при назначении амиодарона. Таким образом, раннее назначение антиаритмической терапии способствовало восстановлению сократительной функции предсердий и препятствовало развитию их «аритмогенного» ремоделирования.

\section{Заключение}

У больных ИБС с персистирующей формой ФП в первые сутки после восстановления СР обнаружены расширение полости ЛП со снижением его сократительной функции и диастолическая дисфункция ЛЖ. При этом у больных ИБС с персистирующей формой ФП достижение целевых значений ЧЖС на протяжении 6 мес лечения бисопрололом способствовало улучшению показателей диастолической функции миокарда ЛЖ, но не влияло на размер и сократительную функцию ЛП. Сохранение синусового ритма в течение 6 мес лечения амиодароном у больных ИБС с персистирующей формой ФП способствовало уменьшению размера ЛП, восстановлению его сократимости и улучшению показателей диастолической функции миокарда Лж.

Конфликт интересов. Все авторы заявляют об отсутствии потенциального конфликта интересов, требующего раскрытия в данной статье.

\section{References / Литература}

1. Camm A. J. Atrial fibrillation and risk. ClinCardiol 2012; 35 Suppl 1: 1-2

2. Guidelines for the management of atrial fibrillation. The Task Force for the Management of Atrial Fibrillation of the European Society of Cardiology (ESC). Eur Heart J 2010; 31(19):2369-429.

3. AFFIRM Investigators. A comparison of rate control and rhythm control in patients with atrial fibrillation. N Engl J Med 2002;347:1825-33.

4. Van Gelder IC, Hagens VE, Bosker HA et all. A comparison of rate control and rhythm control in patients with recurrent persistent atrial fibrillation. N Engl J Med 2002;347:1834-40.

5. Carlsson J, Miketic S, Windeler J and the STAF Investigators. Randomized trial of rate-control versus rhythmcontrol in persistent atrial fibrillation. J Am CollCardiol 2003;41:1690-6

6. Opolski G, Torbicki A, Kosior DA Rate control vs rhythm control in patients with nonvalvular persistent atrial fibrillation: the results of the Polish How to Treat Chronic Atrial Fibrillation (HOT CAFE) Study. Chest 2004; 126:476-86

7. Roy D, Talajic M, Nattel S, et all. Rhythm control versus rate control for atrial fibrillation and heart failure. N Engl J Med 2008;358:2667-77.

8. Hohnloser SH, Kuck KH, Lilienthal Jet all. Rhythm or rate control in atrial fibrillation-Pharmacological Intervention in Atrial Fibrillation (PIAF): a randomised trial. Lancet 2000;356:1789-94.

9. Camm A. J., Savelieva I. Atrial fibrillation: the rate versus rhythm management controversy. J R Coll Physicians Edinb 2012; 42 Suppl 18: 23-34.

10. Boldt A, Wetzel U, Weigl J, et al. Expression of angiotensin II receptors in human left and right atria tissue in atrial fibrillation with and without underlying mitral valve disease. J Am Coll Cardiol 2003:42:1785-92

11. Goette A, Arndt M, Rocken C, et al. Regulation of angiotensin II receptor subtypes during atrial fibrillation in humans. Circulation 2000;101:2678-81.

12. Oudot A, Vergely C, Ecarnot-Laubriet A, et al. Angiotensin II activates NADPH oxidase in isolated rat hearts subjected to ischaemia-reperfusion. Eur J Pharmacol 2003;462:145-54

13. Gronefeld GC, Hohnloser SH. Heart failure complicated by atrial fibrillation: mechanistic, prognostic, and therapeutic implications. J Cardiovasc Pharmacol Ther 2003; 8(2): 107-13.

14. Caldeira D, David C, Sampaio C. Rate versus rhythm control in atrial fibrillation and clinical outcomes: updated systematic review and meta-analysis of randomized controlled trials. Arch Cardiovasc Dis 2012; 105: 226-38. 